REVISTA PROYECCIONES N० 11: 61-79

Julio 1986 - I.S.S.N. 0716-0917

\title{
CONSTRUCCION DE UNA MATRIZ A PARTIR DE SUS AUTOVALORES
}

RICARDO SOTO M.

\section{INTRODUCCION.}

El propósito de este artículo es presentar dos algoritmos, uno debido a Hochstadt [3] y el otro a Boley y Golub [1], los cuales permiten construir una matriz con estructura de banda a partir de sus autovalores. En términos muy generales, el problema es: dado un conjunto de autovalores $\lambda_{i}$, los cuales se supone corresponden a una cierta matriz A, encontrar A. Por supuesto, existen ciertos requerimientos que deben ser safisfechos en orden a encontrar una solución.

Empezaremos por decir algo que intente ser una respuesta, aunque parcial, a las siguientes dos preguntas: ¿cuál es el problema? y ¿ipor qué estudiar este problema? Hemos dicho que el problema es determinar una matriz A, conocido su espectro; pero existen varias específicas versiones de

* Departamento de Matemáticas, Facultad de Ciencias - Universidad del Nor te, Chile. 
él. Algunas son:

a) Construir una matriz de Jacobi, dado su espectro. Este problema ha si do estudiado por Hochstadt (1974) y otros y será visto en detalle en la sección 2 .

b) Determinar una matriz de banda, dado su espectro. Boley y Golub han propuesto un algoritmo (1977) que será presentado en la sección 3.

c) Inverse additive and mulciplicative eigenvalue problems. Entre otros, este problema ha sido estudiado por Friedland (1975) y Biegler-Köning (1981). El problema es: dadas n matrices complejas A y n números complejos $\lambda_{i}$, encontrar n números complejos $c_{i}$ tal que la matriz

$$
A=A_{0}+\sum_{i=1}^{n} c_{i} A_{i} \text { tenga autovalores } \lambda_{1}, \lambda_{2}, \ldots, \lambda_{n} \text {. }
$$

El caso particular en que $A_{i}=e_{i} e_{i}^{T} ; i=1, \ldots, n$ donde $e_{i}$ es el i-ésimo vector unitario es llamado "inverse additive eigenvalue problem" (IAEP) mientras que el caso $A_{0}=0, A_{i}=r_{i} e_{i}^{T}, i=1, \ldots n_{i} r_{i}$ un vector con entradas complejas, es llamado "inverse multiplicative eigen value problem".

Intentando dar una respuesta a nuestra segunda pregunta, mencionaremos que el problema "IAEP" surge en la solución de problemas de SturmLiouville (inverso), es decir, en problemas como el siguiente:

Dado

$$
-u^{\prime \prime}(x)+p(x) u(x)=\lambda u(x) ; x \in[0, \text { II }]
$$

$$
u(0)=u(I)=0
$$

y si suponemos que $\mathrm{p}(\mathrm{x})$ no es conocida, pero sí lo es el espectro $\left\{\lambda_{i}\right\}$ ccó mo podemos determinar $\mathrm{p}(\mathrm{x})$ ?

Discretizar el problema (1.1) usando diferencias finitas lleva a

$$
\frac{1}{h^{2}}\left(-u_{i+1}+2 u_{i}-u_{i-1}\right)+p_{i} u_{i}=\dot{\lambda}_{i} u_{i} ; i=1, \ldots, n
$$




$$
u_{0}=u_{n+1}=0
$$

donde $h=\frac{\Pi}{n+1}, u_{i}=u(i h), p_{i}=p(i h)$.

Ahora (1.2) puede ser escrito en forma matricial como

$$
\left(A_{0}+D\right) u=\lambda u, \text { donde } D=\operatorname{diag}\left\{\mathrm{p}_{i}\right\}
$$

$A_{0}=\frac{1}{h^{2}}\left(\begin{array}{rrrrr}2 & -1 & & & 0 \\ -1 & 2 & -1 & \\ & \ddots & \ddots & \ddots & -1 \\ 0 & & \ddots & \ddots & 2\end{array}\right)$

$u=\left(u_{1}, \ldots u_{n}\right)^{T} ; \lambda=\left(\lambda_{1}, \ldots \lambda_{n}\right)^{T}$

El problema es entonces: dato $A_{0} y$ un espectro $\left\{\lambda_{i}\right\}, i=1 \ldots n$, encontrar una matriz diagonal $\Delta$ tal que $A_{0}+D$ tenga el espectro dado. Es to equivale a tener un "IAEP", el cual puede ser escrito en la forma

$$
A=A_{0}+\sum_{i=1}^{n} p_{i} A_{i} \quad \text { con } A_{i}=e_{i} e_{i}^{T}
$$

2. CONSTRUCCION DE UNA MATRIZ DE JACOBI A PARTIR DE SU ESPECTRO.

Existe concenso entre los matemáticos dedicados al estudio de es te problema (o del problema más general INVERSE FIGENVALUE PROBLEM) en definir una matriz de Jacobi como una matriz nxn tridiagonal, real y simétri ca con entradas ( $i, i+1)$ e (i, $i-1)$ positivas, es decir, una matriz de la forma 


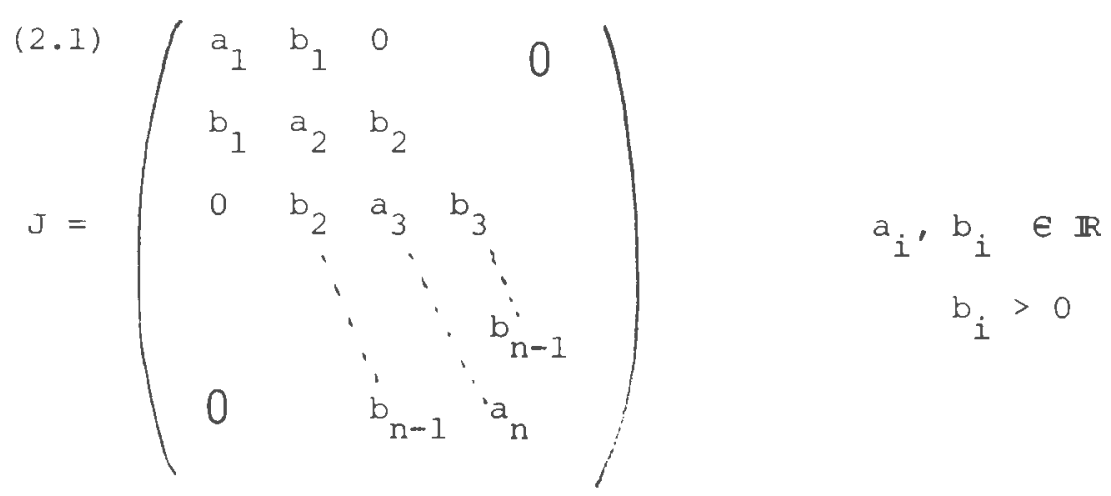

Sea $J_{2}$ la submatriz $(n-1) x(n-1)$, obtenida de J eliminando en ella la primera fila y primera columna y $\operatorname{sean}\left\{\lambda_{i}\right\}$ los autovalores de $J y\left\{u_{i}\right\}$ los autovalores de $\mathrm{J}_{2}$, todos los cuales son reales puesto que $\mathrm{J}_{\mathrm{y}} \mathrm{J}_{2}$ son autoadjuntas.

Es sabido que los autovalores de una matriz de Jacobi son distin tos y puede ser probado que los autovalores $\mu_{i}$ intercalan los autovalores $\lambda_{i}$. Entonces si indicamos los autovalores de modo que $\lambda_{i}>\lambda_{i+1} y$ $\mu_{i}>\mu_{i+1}$ tendremos que

$(2.2)$

$$
\lambda_{1}>\mu_{2}>\lambda_{2}>\mu_{3}>\ldots .>\mu_{n}>\lambda_{n}
$$

Con estas hipótesis podemos establecer el siguiente teorema, debido a Nochstadt (1974), el cual no es un teorema de existencia. El supone a priori que los $\lambda_{i} y \mu_{i}$ son los autovalores de al menos una matriz de Jacobi y asegura que ellos corresponden a precisamente una tal matriz. El teorema da también un algoritmo para la construcción de esa matriz [3].

Teorema (Hochstadt)

$\operatorname{Sean}\left\{\lambda_{i}\right\}$ los autovalores de una matriz de Jacobi $J$ y $\left\{\mu_{i}\right\}$ los autovalores de la submatriz $J_{2}$, satisfaciendo las condiciones ${ }_{i}>\mu_{i+1}>\lambda_{i+1}, \quad i=1,2, \ldots n-1$. Entonces, a partir de $\left\{\lambda_{i}\right\} y\left\{H_{i}\right\}$ una única matriz de Jacobi $J$ puede ser construida. 
Demostración.

Obsérvese que el número de incógnitas (los $a_{i} y$ los $b_{i}$ ) es igual al número de autovalores dados $\left(\lambda_{i} y u_{i}\right)$.

Consideremos la equación matricial

$$
(\lambda I-J) v=e_{1}, \text { donde }
$$

$\lambda$ no es un autovalor y $e_{1}=(1,0, \ldots 0)$. Mediante el uso de la regla de Cramer, es fácil ver que la primera entrada del vector v, la cual puede ser escrita en la forma $\left(v, e_{1}\right)$, está dada por

$$
\left(v, e_{1}\right)=\frac{\prod_{i=2}^{n}\left(\lambda-\mu_{i}\right)}{\prod_{i=1}^{n}\left(\lambda-\lambda_{i}\right)}=\frac{\operatorname{det}\left(\lambda I-J_{2}\right)}{\operatorname{det}(\lambda I-J)}=\frac{P(\lambda)}{Q(\lambda)},
$$

donde $P(\lambda)$ y $Q(\lambda)$ son los polınomios característicos de $J_{2}$ y $\mathrm{J}$ respectivamente.

De (2.3) vemos que el vector $\mathrm{v}$ puede ser expresado como

$$
\mathrm{v}=(\lambda I-J)^{-1} \mathrm{e}_{1} \quad(\lambda \text { no es autovalor de } \mathrm{J})
$$

Entonces para $|\lambda|>\max \left|\lambda_{i}\right|$ tenemos, usando una expansión de Newmann,

$$
\begin{aligned}
(\lambda I-J)^{-1} & =\left(\lambda\left(I-\frac{1}{\lambda} J\right)\right)^{-1}=\frac{1}{\lambda} \sum_{k=0}^{\infty} \frac{1}{k} J^{k}, \text { de donde } \\
v & =\frac{1}{\lambda} \sum_{k=0}^{\infty} \frac{1}{\lambda^{k}} J^{k} e_{1} \quad y \\
\left(v, e_{1}\right) & =\frac{1}{\lambda} \sum_{k=0}^{\infty} \frac{1}{\lambda^{k}}\left(J^{k} e_{1}, e_{1}\right) .
\end{aligned}
$$

Consideremos ahora el lado derecho de (2.4), al cual podemos 
aplicar una descomposición en fracciones parciales para obtener

$$
R(\lambda)=\frac{P(\lambda)}{Q(\lambda)}=\sum_{i=1}^{n} \frac{A_{i}}{\lambda-\lambda_{i}} \quad \text {, donde los coeficientes } A_{i} \text { son de- }
$$

terminados como los residuos de $R(\lambda)$ en los polos $\lambda_{i}$, integrando alrededor de un círculo $C_{i}$ de radio suficientemente pequeño. Esto lleva a

$$
A_{i}=\operatorname{Res}_{\lambda=\lambda_{i}} R(\lambda)=\frac{P\left(\lambda_{i}\right)}{Q^{\prime}\left(\lambda_{i}\right)} \quad, \quad \text { de donde }
$$

$$
\frac{P(\lambda)}{Q(\lambda)}=\sum_{i=1}^{n} \frac{P\left(\lambda_{i}\right)}{Q^{\prime}\left(\lambda_{i}\right)\left(\lambda-\lambda_{i}\right)}
$$

Para $|\lambda|>\max \left|\lambda_{i}\right|$, tenemos que

$$
\frac{P\left(\lambda_{i}\right)}{Q^{\prime}\left(\lambda_{i}\right)\left(\lambda-\lambda_{i}\right)}=\frac{P\left(\lambda_{i}\right)}{Q^{\prime}\left(\lambda_{i}\right)} \frac{1}{\lambda} \sum_{k=0}^{\infty}\left(\frac{\lambda i}{\lambda}\right)^{k}, \text { lo cual lleva a }
$$

$$
\frac{P(\lambda)}{Q(\lambda)}=\frac{1}{\lambda} \quad \sum_{=0}^{\infty} \frac{1}{\lambda^{k}} \sum_{i=1}^{n} \frac{\lambda_{i}^{k} P\left(\lambda_{i}\right)}{Q^{\prime}\left(\lambda_{i}\right)}
$$

De (2.4) tenemos que $\left(v, e_{1}\right)=\frac{P(\lambda)}{Q(\lambda)}$. Entonces por (2.7) y (2.9) obtenemos

$$
\left(J^{k} e_{1}, e_{1}\right)=\sum_{i=1}^{n} \frac{\lambda_{i}^{k} P\left(\lambda_{i}\right)}{Q^{\prime}\left(\lambda_{i}\right)} ; k=0,1,2, \ldots
$$

En particular para $k=1$ vemos que

$$
\left(J e_{1}, e_{1}\right)=a_{1}=\sum_{i=1}^{n} \frac{\lambda_{i} P\left(\lambda_{i}\right)}{Q^{\prime}\left(\lambda_{i}\right)}
$$

$$
\text { Fara } k=2 \text { : }
$$

$$
\left(J^{2} e_{1}, e_{1}\right)=a_{1}^{2}+b_{1}^{2}=\sum_{i=1}^{n} \frac{\lambda_{i}^{2} P\left(\lambda_{i}\right)}{Q^{\prime}\left(\lambda_{i}\right)}
$$


Puesto que $a_{1}$ es conocido de (2.11) y $b_{1}>0$, entonces (2.12) da $\mathrm{b}_{1}$ univocamente. Así, hemos determinado la primera fila de J. Supongamos ahora, que las primeras $\mathrm{k}-1$ filas de $\mathrm{J}$ han sido ya determinadas. Entonces tendremos que

$$
\mathrm{J}^{\mathrm{k}-1} e_{1}=\mathrm{d}_{1} e_{1}+\mathrm{d}_{2} e_{2}+\ldots .+\mathrm{d}_{\mathrm{k}} \mathrm{e}_{\mathrm{k}}, \text { donde }
$$

los coeficientes $\left\{a_{j}\right\}$ dependen de $a_{1}, a_{2}, \ldots a_{k-1}$ y $b_{1}, b_{2}, \ldots . b_{k-1} y$ son por 10 tanto conocidos. Además es fácil ver que $d_{k}=b_{1}, b_{2}, \ldots, b_{k-1}$, el cual es positivo puesto que cada $b_{i}$ lo es. En efecto, si $k=2$ tenemos $\mathrm{d}_{\mathrm{k}}=\mathrm{b}_{1}$ mientras que para $\mathrm{k}=3, \mathrm{~d}_{3}$ es el producto escalar $\left(0 b_{2} a_{3} b_{3} 0 \ldots 0\right) \cdot\left(a_{1} b_{1} 0 \ldots 0\right)^{T}=b_{1} b_{2}$. En general $d_{k}$ es el producto escalar entre la $(k+1)$-ésima fila de $J$ y la primera columna de $\mathrm{J}^{\mathrm{k}-1}$.

Aplicando $J$ a $e_{k}$ tendremos la k-ésima columna de $J$, la que puede ser escrita como

$$
J e_{k}=b_{k-1} e_{k-1}+a_{k} e_{k}+b_{k} e_{k+1} \text {. }
$$

Entonces, de (2.13) y (2.14) encontramos que

$$
\begin{aligned}
J^{k} e_{1} & =J J^{k-1} e_{1}=d_{1} J e_{1}+\ldots .+d_{k} J e_{k} \\
& =d_{k} b_{k} e_{k+1}+\left(d_{k} a_{k}+d_{k-1} b_{k-1}\right) e_{k}+T, \text { donde } T \text { indica }
\end{aligned}
$$

los términos que involucran $e_{k-1}, e_{k-2}, \ldots e_{1} \cdot$ Así,

$$
\begin{aligned}
\left(J^{2 k-1} e_{1}, e_{1}\right) & =\left(J^{k-1} e_{1}, J^{k} e_{1}\right)=d_{k}^{2} a_{k}+\ldots . \\
& =\sum_{i=1}^{n} \frac{\lambda_{i}^{2 k-1} P\left(\lambda_{i}\right)}{Q^{\prime}\left(\lambda_{i}\right)}
\end{aligned}
$$

donde los términos indicados por puntos dependen de $a_{1}, \ldots a_{k-1}$ y 
$b_{1}, \ldots b_{k-1}$. Por lo tanto $(2.16)$ determina $a_{k}$. Del mismo modo,

$$
\begin{aligned}
\left(J^{2 k} e_{1}, e_{1}\right) & =\left(J^{k} e_{1}, J^{k} e_{1}\right)=d_{k}^{2}\left(a_{k}^{2}+b_{k}^{2}\right)+\ldots . \\
& =\sum_{i=1}^{n} \frac{\lambda_{i}^{2 k} P\left(\lambda_{i}\right)}{Q^{\prime}\left(\lambda_{i}\right)}
\end{aligned}
$$

donde los términos indicados por puntos dependen de $a_{i} y b_{i}$ como antes. Así, (2.17) determina $b_{i} y$ puesto que las primeras $k$ filas de $J$ han sido encontradas, todas sus filas pueden ser computadas. Esta construcción lle va a una única matriz J. Si hibiera otra matriz M con los mismos autovalo res dados, entonces $(2.10)$ implicaría que

$$
\left(J^{k} e_{1}, e_{1}\right)=\left(M^{k} e_{1}, e_{1}\right), \quad K=0,1,2, \ldots
$$

y por el uso del algoritmo descrito en la demostración del teorema conclui ríamos que $\mathrm{J}=\mathrm{M}$.

Hochstadt también demostró en [3], que la matriz J depende con tinuamente de los autovalores $\lambda_{i}$ de $J$ y $u_{i}$ de $J_{2} y$ además que bajo espe ciales condiciones de simetría sobre los elementos de la matriz J, $a_{i}=a_{n+1-i}$ y $b_{i}=b_{n-i}$, el espectro $\left\{\lambda_{i}\right\}$ de $J$ es suficiente para construir la matriz J univocamente mediante el procedimiento propuesto en el teorema anterior.

Para sentir más concretamente el algoritmo de Hochstadt, conside remos el siguiente pequeño ejemplo:

Dados los autovalores $\lambda_{1}=2+\sqrt{2}, \lambda_{2}=2, \lambda_{3}=2-\sqrt{2}$ correspondientes a una matriz de Jacobi J y los autovalores $\mu_{2}=3, \mu_{3}=1$ de la submatriz $\mathrm{J}_{2}$, encontrar $\mathrm{J}$.

$$
\text { Puesto que } \frac{P\left(\lambda_{i}\right)}{Q^{\prime}\left(\lambda_{i}\right)}=\frac{P\left(\lambda_{3}\right)}{Q^{\prime}\left(\lambda_{3}\right)}=0.25 \quad y \quad \frac{P\left(\lambda_{2}\right)}{Q^{\prime}\left(\lambda_{2}\right)}=0.5
$$


tenemos de (2.11) $a_{1}=2 \quad y$ de (2.12)

$$
a_{1}^{2}+b_{1}^{2}=5 \text { lo cual da } \quad b_{1}=1
$$

Ahora, de (2.16) obtenemos $\mathrm{a}_{2}$ :

$$
\left(J^{3} e_{1}, e_{1}\right)=a_{1}^{3}+2 a_{1} b_{1}^{2}+a_{2} b_{1}^{2}=14 \text {, es decir, } a_{2}=2 \text {. }
$$

De (2.17) $\quad a_{1}^{4}+3 a_{1}^{2} b_{1}^{2}+2 a_{1} a_{2} b_{1}^{2}+a_{2}^{2} b_{1}^{2}+b_{1}^{2} b_{2}^{2}+b_{1}^{4}=41.999999$.

Entonces $\mathrm{b}_{2}=0.999999$. Finalmente $\mathrm{a}_{3}$ es obtenido de $(2.16)$ como $\mathrm{a}_{3}=2$. Por lo tanto nuestra matriz $J$ es

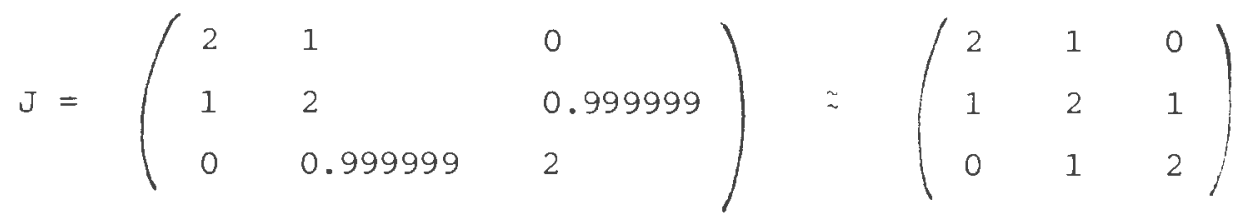

En efecto, los autovalores de la matriz de la derecha están dados por

$$
\lambda_{j}=2+2 \cos \frac{j \Pi}{4}=\{2+\sqrt{2}, 2,2-\sqrt{2}\}
$$

\section{CONSTRUCCION DE UNA MATRIZ BANDEADA A PARTIR DE SU ESPECTRO.}

En esta sección describiremos un algoritmo presentado por D. Boley y G.A. Golub en [1], el cual trata en particular con la determinación de una matriz pentadiagonal a partir de 3 conjuntos de autovalores. En el caso general de una matriz $(2 \mathrm{p}-1)$ diagonal, nuestras hipótesis serán:

Sea A una matriz real simétrica tal que $a_{i j}=0$ si $|i-j| \gtreqless p$. Definimos las submatrices $A^{(k)}(i, j)=\left(a_{i j}\right)$ para $i, j=k, k+1, \ldots, n$ y denotamos los autovalores 
de $A^{(k)}$ por $\lambda_{i}^{(k)}, i=1,2, \ldots, n-k+1$, los cuales suponemos satisfacer las siguientes condiciones

$$
\lambda_{i}^{(k)}<\lambda_{i+1}^{(k)} \quad y \quad \lambda_{i}^{(k)}<\lambda_{i}^{(k+1)}<\lambda_{i+1}^{(k)} ; i=1,2, \ldots n-k .
$$

El problema es entonces determinar A a partir de p conjuntos de autovalores $\lambda_{i}^{(k)}, i=1,2, \ldots n-k+1, d=1,2, \ldots p$. El caso $p=2$ corresponde a determinar una matriz tridiagonal.

Consideraremos aquí entonces $\mathrm{p}=3$ y $\mathrm{n}$ un número par. Sean $K=A, I=A(2)$ y $M=A{ }^{(3)}$ con autovalores $\left\{k_{i}\right\} \quad i=1, \ldots, \quad\left\{\lambda_{i}\right\}$, $i=1, \ldots$ n-1 y $\left\{\mu_{i}\right\} \quad i=1, \ldots n-2$, respectivamente. De (3.1) tenemos que

$$
k_{i}<\lambda_{i}<k_{i+1} ; \lambda_{i}<\mu_{i}<\lambda_{i+1} ; \mu_{i}<\mu_{i+1}
$$

3.1 Empezaremos por considerar la solución de un problema complementario y relacionado al que nos preocupa. Este problema se refiere a encon trar los valores estacionarios de una cierta forma cuadrática sujeta a ciertas restricciones. En particular queremos determinar un vector unitario $\mathrm{C}$ tal que los valores estacionarios de

(3.3) $\quad x^{T} A x$, sujetos a las restricciones.

(3.4) $x^{T} x=1$ y $C^{T} x=0$, sean los valores $\beta_{i}, i=1, \ldots n-1$ tales que $\beta_{i}<\beta_{i+1}$ y $\alpha_{i}<\beta_{i}<\alpha_{i+1}$ donde $\alpha_{i}$ son los autovalores (distintos) de la matriz A.

Sea entonces,

(3.5) $\quad L(x, \lambda, \mu)=x^{T} A x-\lambda\left(x^{T} x-1\right)+2 \mu x^{T} C$,

donde $\lambda y$ H son aquí, multiplicadores de Lagrange. Luego tenemos

$$
\nabla L(x, \lambda, \mu)=2 A x-2 \lambda x+2 \mu C=0,
$$


de donde

(3.7) $\quad x=-\mu(A-\lambda I)^{-1} C$

Y

$$
C^{T} x=-\mu C^{T}(A-\lambda I)^{-1} C=0
$$

Sea $\mu \neq 0$ y $A=Q \Lambda Q^{T}$, donde $\Lambda$ es la matriz diagonal de los autovalores de A Y $Q$ es la matriz de los autovectores de A (normalizados). Entonces sustituyendo en (3.8) tenemos

$$
\begin{aligned}
& C^{\mathrm{T}}\left(Q \Lambda Q^{\mathrm{T}}-\lambda Q Q^{\mathrm{T}}\right)^{-1} C=0 \\
& C^{\mathrm{T}} Q(\Lambda-\lambda I)^{-1} Q^{\mathrm{T}} \mathrm{C}=0
\end{aligned}
$$

Poniendo $\mathrm{d}=\mathrm{Q}^{\mathrm{T}} \mathrm{C}$, obtenemos $\mathrm{d}^{\mathrm{T}}(\Lambda-\lambda I)^{-1} \mathrm{~d}=0 \quad 0$ equivalentemente

$$
\sum_{i=1}^{n} \frac{d_{i}^{2}}{\alpha_{i}-\lambda}=0 \quad \text { con }
$$

$\sum_{i=1}^{n} d_{i}^{2}=d^{T} d=c^{T} Q Q^{T} C=C^{T} C=1$

$$
\text { Poner } \lambda=B_{j}, j=1,2, \ldots n^{-1} \text { en (3.9) lleva a un sistema de }
$$

ecuaciones lineales que define los $d_{i}^{2}$. En orden a determinar una explicita solución para este sistema definamos el polinomio característico

(3.10) $\phi(\lambda)=\underset{j=1}{n-1}\left(\beta_{j}-\lambda\right)$

El miembro izquierdo de (3.9) puede ser expresado como un polínomio escribiendo

$$
\psi(\lambda)=\prod_{j=1}^{n}\left(\alpha_{j}-\lambda\right) \sum_{i=1}^{n} \frac{d_{i}^{2}}{\alpha_{i}-\lambda}
$$


o bien

$$
\psi(\lambda)=\sum_{i=1}^{n} d_{i}^{2} \prod_{\substack{j \neq 1 \\ j \neq i}}^{n}\left(\alpha_{j}-\lambda\right)
$$

Puesto que queremos computar $d$, con $d^{\mathrm{T}} \mathrm{d}=1$, tal que $\psi(\lambda)=\phi(\lambda)$, entonces ponemos $\psi\left(\alpha_{k}\right)=\phi\left(\alpha_{k}\right)$ para $k=1, \ldots, n$. Esto 1leva a

$$
d_{k}^{2}=\frac{\sum_{\substack{n=1 \\ j \neq 1 \\ j \neq k}}^{n-1}\left(\alpha_{j}-\alpha_{k}\right)}{j \neq k}
$$

La condición $a_{i}<\beta_{i}<\alpha_{i+1}$ garantiza que el miembro derecho en (3.13) es positivo. Por otra parte, podemos asignar a $d_{k}$ un valor positivo 0 negativo, de suerte que podríamos encontrar $2^{\mathrm{n}}$ diferentes soluciones. observemos también que $d_{k}$ depende sólo de los "i $y \dot{k}_{i}$, los cuales son dados y una vez que el vector d ha sido calculado, el cómputo del već tor $\mathrm{C}$ es directo de $\mathrm{C}=\mathrm{Qd}$.

Finalmente, digamos que si los autovalores y valores estacionarios son dados $y$ el vector $C$ es especificado, entonces podemos deducir información acerca de la matriz de autovectores. En particular si $C=e_{1}=(1,0 \ldots 0)^{\mathrm{T}}$, entonces la primera fila de la matriz $\mathrm{Q}$ de autovectores es determinada. En efecto, puesto que la primera culumna de $Q^{T}$ es la primera fila de $Q$, se tiene

$$
\mathrm{Q}^{\mathrm{T}} \mathrm{C}=\mathrm{d} \Rightarrow \mathrm{Q}^{\mathrm{T}} \mathrm{e}_{1}=\mathrm{d} \Rightarrow \mathrm{Q}^{1}=\mathrm{d}, \text { donde } \mathrm{Q}^{1} \text { es la primera fila }
$$

de Q. Entonces

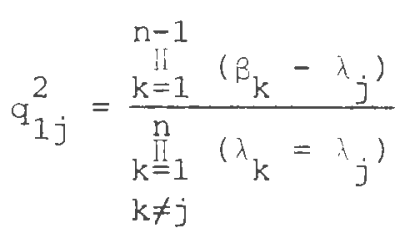

Si $C=e_{1}$, entonces los valores estacionarios de $x^{\mathrm{T}} \mathrm{Ax}$ sujetos a 
las restricciones (3.4) son los autovalores de la submatriz A ${ }^{(2)}$ obtenido de A eliminando en ella la primera fila y columna.

\section{Tenemos entonces el siguiente lema:}

Lema 3.1. Sea A una matriz real simétrica con autovalores distintos $\lambda_{i}^{(1)}, i=1, \ldots n$ y sea $A{ }^{(2)}$ la submatriz obtenida de $A$ como arriba con autovalores $\lambda_{i}^{(2)}, i=1, \ldots n-1$. Si $A=Q \Lambda Q^{T}$, donde $Q$ es la matriz de autovectores de $A$, entonces

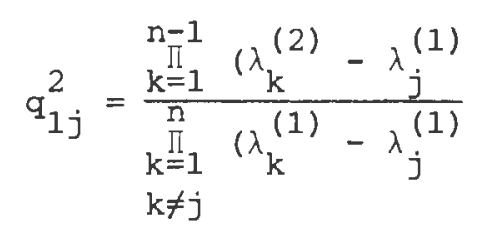

\subsection{El Algoritmo de Lanczos en bloques.}

La idea en el algoritmo de Lanczos es generar a partir de una ma triz simétrica dada A una matriz tridiagonal $T$, la cual tiene los mismos autovalores que A. Así dada A, queremos determinar las columnas de una ma triz $x$ tal que

(3.16) $\quad X^{T} A X=T \quad$ con $\quad X^{T} X=T$

Ahora, en particular, deseamos que T tenga una forma tridiagonal en bloques

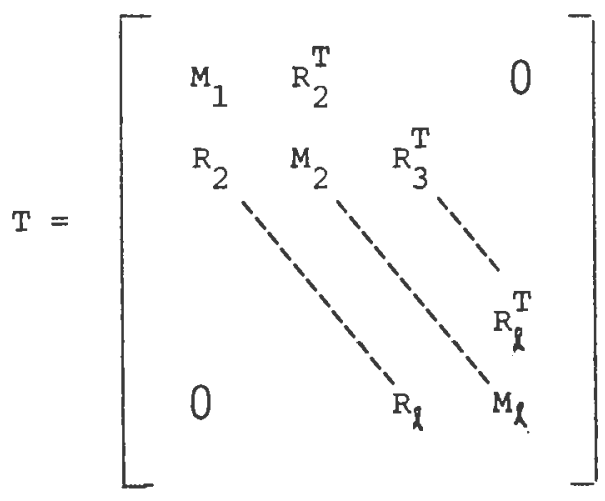


donde las matrices $M_{i}$ y $R_{i}$ son de orden $\operatorname{cxr}$ con $r l=n$ y $R_{i}$ es una matriz sobretriangular. Con estas condiciones $T$ resulta ser pentadiagonal. Para ver esto sea $n=6$ y $\quad r=2$ (entonces $l=3$ ):

$T=\left[\begin{array}{cc|cc|cc}x & x & x & 0 & 0 & 0 \\ x & x & x & x & 0 & 0 \\ \hline x & x & x & x & x & 0 \\ 0 & x & x & x & x & x \\ \hline 0 & 0 & x & x & x & x \\ 0 & 0 & 0 & x & x & x\end{array}\right]$

De (3.16) tenemos que $\mathrm{AX}=\mathrm{XT}$ y con una adecuada partición de la matriz $\mathrm{X}$ obtenemos

$$
A x_{1}=X_{1} M_{1}+X_{2} R_{2}
$$

$$
A x_{i}=x_{i-1} R_{i}^{T}+x_{i} M_{i}+x_{i+1} R_{i+1}
$$

donde $x_{i}$ es una matriz nxr.

Dada $x_{1}$ nxr tal que $x_{1}^{T} x_{1}-I_{r}$, lit i lea es computar una sucesión de matrices $x_{2}, \ldots x_{l}, M_{1}, \ldots M_{k} y k_{2}, \ldots R_{A}$. Así, el algoritmo puede ser descrito como

$1^{\circ}$ Computar $\mathrm{Ax}_{1}$ y $\mathrm{M}_{1}=\mathrm{X}_{1}^{\mathrm{T}} \mathrm{AX}_{1}$

$2^{\circ} \quad$ Para $i=2,3, \ldots, t-1$

a) Computar $z_{2}=\mathrm{AX}_{1}-\mathrm{X}_{1} \mathrm{M}_{1}=\mathrm{X}_{2} \mathrm{R}_{2}$

$$
z_{i+1}=A X_{i}-x_{i} M_{i}-X_{i-1} F_{i}^{T}=x_{i+1} F_{1+1}
$$

b) Computar $x_{i+1}$ y $R_{i+1}$ tales que $x_{i+1}^{T} x_{i+1}-I_{r}$ y $R_{i+1}$ 


$$
\begin{aligned}
& \text { es sobretriangular con } z_{i+1}=x_{i+1} R_{i+1} \\
& \text { c) Computar } \quad x_{i+1} \text { Y } M_{i+1}=x_{i+1}^{T} A x_{i+1} \text {. }
\end{aligned}
$$

El paso $2^{\circ}$ b) puede ser efectuado por medio del proceso de ortogonalización de Gram-Schmidt. Salvo error de redondeo, las matrices $x_{i}$ deberían satisfacer

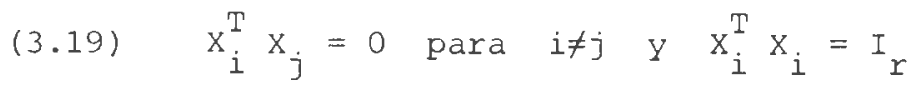

Si $A=Q \Lambda Q^{T}$ donde $\Lambda$ es la matriz diagonal de autovalores de $A$ Y $Q$ la matriz ortogonal de autovectores de $A$, entonces $\therefore=\mathscr{Q}^{\mathrm{T}} \mathrm{AQ}$. Por 10 tanto la matriz $Q$ corresponde a la matriz $\mathrm{x}^{\mathrm{T}}$ y tomando $\mathrm{x}_{1}^{\mathrm{T}}$ igual a las primeras $r$ filas de la matriz $Q$ tenemos que $X^{T}=Q D$ donde $D$ es una matriz diagonal con $\mathrm{d}_{i}= \pm 1$.

Así, si queremos construir una matriz pentadiagonal K=A (para 10 cual r=2), necesitamos computar las primeras dos componentes de los autovectores de $K$. Esto puede ser hecho del siguiente modo: Por uso del lema 3.1 dado en la sección 3.1, podemos calcular los cuadrados de las primeras componentes de los autovectores de $\mathrm{K}$ a partir de los autovalores $\mathrm{K}_{\mathrm{i}} \mathrm{de} K$ y $\lambda_{i}$ de $L=A^{(2)}$ y los cuadrados de las primeras componentes de 10 autovectores de $L$ a partir de los autovalores $t_{i}$ de $L y_{i}$ de $M=A(3)$. Aho ra bien, será mostrado enseguida, que las primeras componentes de los auto vectores de $\mathrm{L}$ determinan las segundas componentes de 1 os autovectores de $\mathrm{K}$. una vez que las primeras dos filas de $Q$ son conocidas, estaremos en condiciones de generar una matriz pentadiagonal por el uso del algoritmo de Lanczos en bloque.

3.3. Construcción de la matriz pentadiagonal.

Particionemos la matriz $\mathrm{K}$ en la siguiente forma: 
$(3.20)$

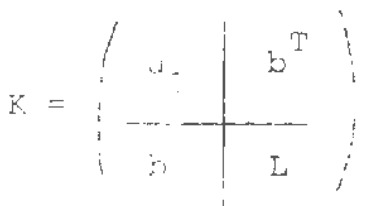

Sean $Q$ la matriz de autovectores de $k$ y ${ }^{2}$ la matriz de autovecto res de L. Entonces

$$
\overrightarrow{k q}_{j}=k_{j} \vec{q}_{j} ; j=1, \ldots \text {. } \quad y \quad L_{\vec{p}}=\vec{j}_{j}, \quad j=1,2, \ldots n-1
$$

Por el lema 3.1 hemos ya computade $1_{1 j} y \mathrm{P}_{j j} \mathrm{y}$ ahora queremos calcular $q_{2 j}$.

Examinemos ahora la relación entre los autovalores de $k$ y $L$ ei vector b. si

$$
\hat{b}=\mathrm{P}^{\mathrm{T}} \mathrm{y} \quad \mathrm{k}_{i} \mathrm{y} \quad \mathrm{i}_{i} \text { estan dados, entonces puede ser }
$$

mostrado que

$(3.22)$

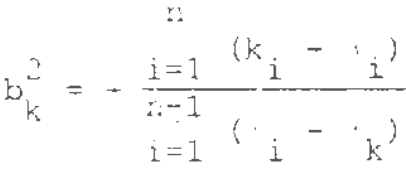

$$
\begin{aligned}
& i \neq k
\end{aligned}
$$

Anors, de $(3.21)$ tenemos ye

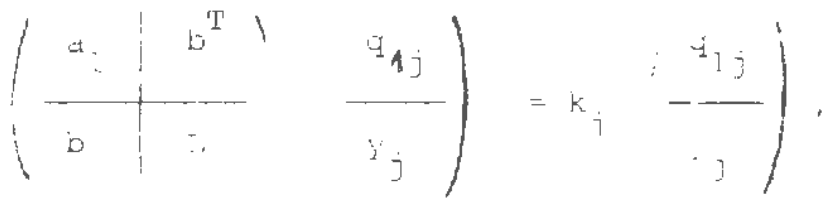

es decir,

$$
\begin{aligned}
& w_{i} q_{1 j}+b^{T} y_{j}-k_{j} q_{l} j \\
& a_{i j} b+L y_{j}=k_{j} y_{j},
\end{aligned}
$$

$(3.23)$

donue es claro que $q_{1 j}$ es un escalar $y y_{j}$ un vector con $n-l$ entradas. 
Ahora (3.23) lleva a

$$
\left(L-k_{j} I\right) y_{j}=-q_{1 j} b
$$

$y$ puesto que $\quad L=P \Gamma P^{T}$, donde $\Gamma=\operatorname{diag}\left\{\lambda_{i} ;\right.$ entonces

$$
\begin{aligned}
& y_{j}=-q_{j j}\left(P \Gamma P^{T}-k_{j} I\right)^{-1} b \\
& y_{j}=-q_{i j} p\left(\Gamma-k_{j} I\right)^{-1} P^{T} b \\
& y_{j}=-q_{i j} p\left(\Gamma-k_{j} I\right)^{-1} b
\end{aligned}
$$

Así tenemos

$$
q_{2 j}=y_{1 j}=-\sum_{k=1}^{n-1} \frac{p_{1 k} b_{k}}{\left(\lambda_{k}-k_{j}\right)} q_{1 j}
$$

Luego el algoritmo (Boley-Golub) puede ser resumido como sigue:

1. computar $q_{1 j}^{2}$ y $p_{1 j}^{2}$ por (3.15)

2. Computar $\mathrm{b}_{j}^{2} \quad$ por $(3.22)$

3. Computar $q_{2 j} \quad$ por $(3.24)$

4. Aplicar el algoritmo de Lanczos en bloque empezando con

$$
x_{1}=\left(\begin{array}{lllll}
q_{11} & q_{12} & \cdots & q_{1 n} \\
q_{21} & q_{22} & \cdots & q_{2 n}
\end{array}\right)^{\top}
$$

Obsérvese que los elementos en las posiciones $(1,1)$ y $(2,2$, son unívocamente determinados puesto que

$$
\mathrm{a}_{1}=\operatorname{traza} \mathrm{k}-\operatorname{traza} \mathrm{L} \quad \mathrm{y} \quad \mathrm{a}_{2}=\operatorname{traza} \mathrm{L}-\operatorname{traza} \mathrm{M} .
$$


Por otra parte, el signo de. producto $p_{1 k} b_{k}$ en (3.24) y el signo de $q_{1 j}$ no están aeterminados, por lo cual diferentes matrices $k$ pueden ser obtenidas.

\section{COMENTARIOS.}

Los dos algoritmos descritos aquí presentan algunas dificultades. En primer lugar, en el algoritmo de lu hstadt para generar una matriz de Jacobi (sección 2), la principal dificultad está, cuizás, en cómo obtener de un modo simple y eficiente los productos interiores en (2.16) y (2.17), es decir, las entradas $(1,1)$ en $J^{2 k-1} y J^{2 k}$, cuando $k$ deja de ser apropiadamente pequeño. A su turno, el algor tmo de Boley-Golub genera un، matriz bandeada que está lejos de ser únisa. Así por ejemplo, para p = 3 (matriz pentadiagonal) el algoritmo puede llevar a $2^{2 \mathrm{n}-1}$ posibles matrices. También es supuesto en este algoritmo jue $(p-1)$ divide a $n$ donde $n$ es un entero par. Obviamente el algoritmo de Boley-Golub puede ser aplicado a construir una matriz de Jacobi.

Nuestra intención es presentur algunos ejemplos y discutir un nue vo algoritmo en una próximá ocasión. 


\section{BIBLIOGRAFIA.}

[1] D. BOLEY and G.H. GOLUB. "Inverse Eigenvalue Problems for band matri ces". Numerical Analysis Proceeding, Biennial Conference, Dundee 1977. Lecture Notes in Mathematics, Springer-Verlag.

[2] G.H. GOLUB. "Some Modified Matrix Eigenvalue Problems", Siam Review, vol. 15, $\mathrm{N}^{\circ} 2,1973$.

[3] H. HOCHSTADT. "On the Construction of a Jacobi Matrix fron Spectral Data", Linear Algebra and its Applications 8, 435-446, 1974.

[4] S. FRIEDLAND. "On Inverse Multiplicative Eigenvalue Problems for Matrices", Linear Algebra and its Applications 12, 127-137, 1975.

[5] J. NOCEDAL and M.L. OVERTON. "Numerical Methods for Solving Inverse Eigenvalue Problems", Numerical Methods Proceedings, Caracas, 1982, Lecture Notes in Mathematics, Springer-Verlag. 